\title{
Baseline Analytical Model for Machine-Type Communications Over 3GPP RACH in LTE-Advanced Networks
}

\author{
Konstantin E. Samouylov ${ }^{1}$, Yuliya V. Gaidamaka ${ }^{1(\bowtie)}$, \\ Irina A. Gudkova ${ }^{1,2}$, Elvira R. Zaripova ${ }^{1}$, and Sergey Ya. Shorgin ${ }^{2}$ \\ 1 Applied Probability and Informatics Department, RUDN University, \\ 6 Miklukho-Maklaya St., Moscow 117198, Russia \\ \{ksam, ygaidamaka, igudkova, ezarip\}@sci.pfu.edu.ru \\ 2 Federal Research Center "Computer Science and Control", Russian Academy \\ of Sciences, 44-2, Vavilova St., Moscow 119333, Russia \\ sshorgin@ipiran.ru
}

\begin{abstract}
Machine-type communication (MTC) is a new service defined by the 3rd Generation Partnership Project (3GPP) to provide machines to interact to each other over future wireless networks. One of the main problems in LTE-advanced networks is the distribution of a limited number of radio resources among enormously increasing number of MTC devices with different traffic characteristics. The radio resources allocation scheme for MTC traffic transmission in LTE networks is also standardized by 3GPP and implements the Random Access Channel (RACH) mechanism for transmitting data units from a plurality of MTC devices. Until now, there is a number of problems with the congestion in radio access network, as evidenced by a series of articles calling attention to the fact that more research is required, and even modification of the RACH mechanism in order to address drawbacks, exhibiting for example when a large number of devices are trying to access simultaneously. However, not many results have been obtained for the analysis, which allows to explore a variety of performance metrics of RACH mechanism on a qualitative level. In this paper the mathematical model in a form of the discrete Markov chain is built taking into account the features of the access procedure under congestion conditions and collisions. This baseline model allows to obtain the solution for key performance measures of RACH mechanism, such as the access success probability and the average access delay, in an analytical closed-form. Based on the proposed baseline model it is possible to obtain new results for the analysis of some modifications of RACH mechanism such as ACB (Access Class Baring).
\end{abstract}

Keywords: LTE-advanced $\cdot$ Machine-type communications $\cdot$ Random access channel $\cdot$ Markov chain $\cdot$ Access success probability $\cdot$ Average access delay

The reported study was funded by RFBR and Moscow city Government according to the research project No. 15-37-70016 mol_a_mos, by RFBR according to the research projects No. 14-07-00090, 15-07-03051, and by Ministry of Education and Science of the Russian Federation (President's Scholarship No. 2987.2016.5). 


\section{Introduction}

In recent years, a huge number of technological devices appeared in the market that support various applications associated with data transfer automatically. In this perspective, a key role will be played by machine-type communications (MTC), which is a new concept where devices exchange data without any (or minimal) human intervention [1]. MTC is expected to open up unprecedented opportunities for telecom operators in the various fields of the new digital economy (home and office security and automation, smart metering and utilities, maintenance, building automation, automotive, healthcare and consumer electronics, etc.), and, therefore, will be one of the economic foundations of emerging $5 \mathrm{G}$ wireless networks [2, 3]. As in the case of any new technology, the analysis of the impact of MTC traffic features requires modification of both classical and modern methods [4-6].

Conventional wireless communication technologies, including 3GPP LTE network, do not allow establishing effectively machine-to-machine (M2 M) connections between a large number of interacting MTC devices. One possible solution of the problem is based on the use of random access (RA) procedure [7, 8]. The advantage of this method is that the MTC devices can access to the radio access channel (RACH), regardless of their arrangement and centralized management.

It is well known that an overload on the RACH level can lead to overload in the entire LTE network. Feature of the M2 M traffic that differs substantially from the traditional $\mathrm{H} 2 \mathrm{H}$ traffic is that existing mechanisms cannot effectively overcome RA procedure overload. MTC devices such as fire detectors usually send small amounts of data periodically while operating in the normal mode. However, in the case of emergency MTC devices generate burst traffic, which can cause overloading [9, 10]. In the case of high network traffic access delay increases significantly, and this can be critical in various emergency situations [7]. Some other features of M2 M traffic transmission were considered in [10-19] taking into account problems of optimal radio resources allocation [11-15], overload control mechanisms based on Access Class Barring (ACB) schemes [10, 14] and other congestion control problems [16, 17].

The purpose of this paper is the analytical modeling of the access procedure which is able to support the simultaneous access of MTC devices. According to [7] the reference scheme of the procedure consists of a four-message handshake between the accessing devices and the base station. In the same 3GPP technical report main measures to RACH capacity evaluation for MTC are specified: collision probability, access success probability, access delay, the number of preamble transmissions to perform a random access procedure successfully, the number of simultaneous preamble transmissions in an access slot.

There are many papers devoted to modeling and simulation of RACH procedure, e.g. interesting results are obtained in [2], which also provides a review of known works on this issue. However, not many analytical models are known, which allow exploring main RACH performance metrics [7] on a qualitative level. We highlight [18], where the formulas for the calculation of these metrics were obtained. Unlike to known results, the objective of this study is to obtain a closed-form solution, which depends on the minimum number of $\mathrm{RACH}$ procedure parameters and is easy for 
calculation. This paper is an extension of [19], where the approach to analytical modeling using Markov chain apparatus was proposed and the Monte Carlo simulation model was developed. In contrast to [19], this paper concentrates on the analytical model of a random access procedure in LTE cell and focuses on two metrics for RACH capacity evaluation - the access success probability and the average access delay in the presence of collisions and physical channel congestion.

The rest of the paper is organized as follows. In Sect. 2 we shortly describe RACH signaling reference scheme, simultaneously discuss notations of the mathematical model and introduce its core assumptions. In Sect. 3, formulas for calculating key metrics in closed form are obtained. Further, in Sect. 4 main performance measures calculating is illustrated via the numerical example. Finally, we conclude the paper in Sect. 5 .

\section{Random Access Procedure, Model Notation and Assumptions}

In this section we consider RACH procedure that is the initial synchronization process between user equipment (UE) and the base station eNB while data exchange performs over Physical RACH (PRACH) in LTE network [7]. Since UEs' attempt for data transmission can be performed randomly and the value of distance to the eNB is unknown, requests for synchronization from various UEs should come with different delays, which is estimated by the level of incoming PRACH signal by eNB.

Widely known RACH procedure defines the sequence of signaling messages transmitted between the UE and the eNB. The procedure begins with a random access preamble transmission to the eNB (Msg 1) by means of one of available PRACH slots (RACH opportunity). The information about slots is broadcasted by the eNB in System Information Block messages. The number of RACH opportunities and the number of preambles depend on the particular LTE network configuration.

After preamble sending the UE waits for a random access response (RAR) (Msg 2) from the eNB within the time interval called a response window. RAR message transmitting over Physical Downlink Control Channel (PDCCH) contains a resource grant for transmission of the subsequent signaling messages. If after the response window is over the UE has not received Msg 2, it means that a collision occurs. The collision of a preamble transmission may occur when two or more UEs select the same preamble and send it at same time slot. In the case of a collision the UE should repeat preamble transmission attempt after a response window. If a preamble collision occurs, the eNB will not send RAR message to all UEs, which have chosen the same preamble. In that case, preambles will be resent after the time interval called the backoff window. If series of collisions occur for a UE after the number of failed attempts exceeds the preamble attempts limit, the RACH procedure is recognized failed.

In the case of successful preamble transmission after receiving Msg 2 from the eNB and RAR processing time, the UE sends connection request (Msg 3) to the eNB using resources of Physical Uplink Shared Channel (PUSCH) [20]. RACH procedure is considered completed after the UE received a contention resolution message (Msg 4) from the eNB. Hybrid automatic repeat request (HARQ) procedure guarantees a 
successful transmission of Msg 3/Msg 4. HARQ procedure provides a limit in Msg 3/Msg 4 sequential transmission attempts. If the limit is reached UE should start a new RACH procedure by sending a preamble.

Making a number of simplifying assumptions for the $\mathrm{RACH}$ procedure, we introduce below the basic notation and build a mathematical model in the form of a discrete Markov chain according to [19]. The time interval between the first RA attempt and the completion of the random access procedure is called an access delay [7]. To analyze this parameter we propose a mathematical model in the form of discrete Markov chain that follows the steps of RACH procedure. The state of the Markov chain determines the number of preamble attempt collisions and the number of sequential Msg 3/Msg 4 transmission attempts. With this model the access delay for each state of the Markov chain can be calculated by summing up the corresponding time intervals introduced below:

$\Delta_{1}^{1}$ - waiting time for a RACH opportunity to transmit a preamble;

$\Delta_{1}^{2}$ - preamble transmission time;

$\Delta_{1}^{3}$ - preamble processing time at the eNB;

$\Delta_{1}^{4}-$ RAR response window;

$\Delta_{1}:=\Delta_{1}^{1}+\Delta_{1}^{2}+\Delta_{1}^{3}+\Delta_{1}^{4}-$ time from the beginning of RACH procedure until sending message Msg 3 or resending a preamble;

$\Delta_{2}$ - backoff window;

$\Delta_{3}-$ RAR processing time;

$\Delta_{4}$ - time for Msg 3 transmission, waiting for Msg 4, and Msg 4 processing.

The model notation is illustrated in message sequence diagram for access success (Fig. 1) and access failure (Fig. 2).

In the case of reliable connections the access delay is equal to the sum of the mentioned above variables $\Delta_{i}, i=1,3,4$. If a collision occurs or connection is

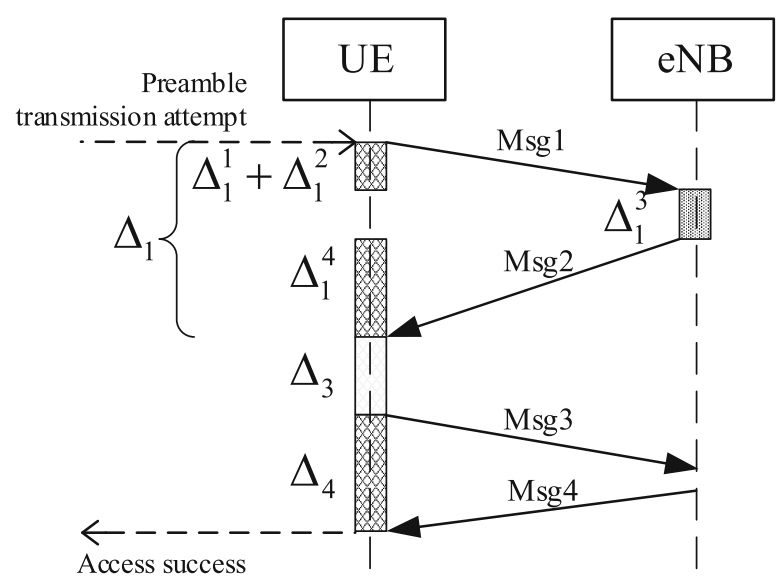

Fig. 1. Message sequence diagram for successful access 

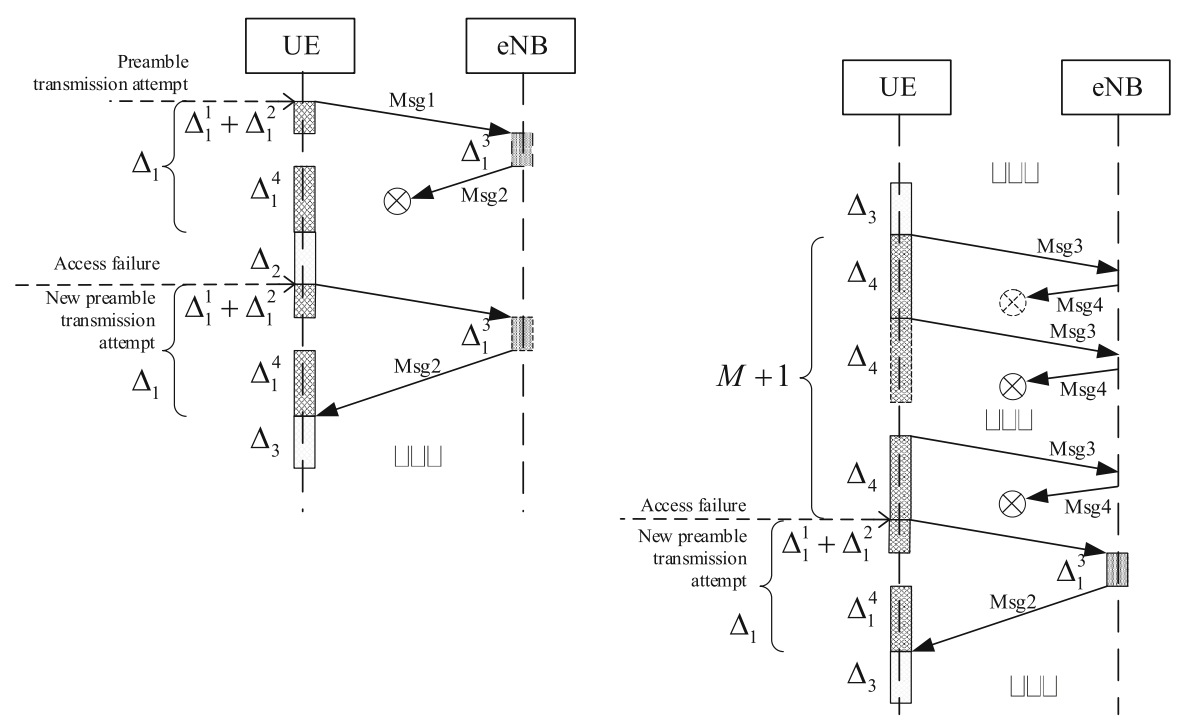

Fig. 2. Message sequence diagram for access failure due to (a) preamble collision (b) contention resolution message retransmission

unreliable the number of retransmissions is limited by $N=9$ for Msg 1 and by $M=4$ for Msg 3 [7]. Let $p$ denote the collision probability, defined as the ratio between the number of occurrences when two or more MTC devices send a random access attempt using exactly the same preamble and the overall number of opportunities (with or without access attempts) in the period [7]. This value depends on the number of MTC devices at eNB coverage area, on intensity $\gamma$ of incoming calls and on LTE network configuration. Also, let $g$ denote the HARQ retransmission probability for Msg 3/Msg 4, and thus we entered all the variables needed further for obtaining formulas for calculation of the access success probability and the average access delay.

\section{The Model and Results in a Closed Form}

The formalization of the above-described RA procedure according to [19] is given by the absorbing discrete-time Markov' chain $\left\{\xi_{i}, i=0, \ldots,(N+1)(M+1)+1\right\}$ with the finite state space

$$
\mathbf{X}=\{(n, m, k), n=0, \ldots, N, m=0, \ldots, M, k=0, \ldots, n\} \cup\{\omega, v\},
$$

initial state $(0,0,0)$, and two absorbing states $\omega$ and $v$. The initial state $(0,0,0)$ represents the beginning of the procedure followed by the first RA attempt, the absorbing state $\omega$ stands for the access success, and the absorbing state $v$ stands for the access failure. Other states denoted by $(n, m, k)$, where $n$ is the number of Msg 1 (preamble) retransmissions, $m$ is the number of Msg 3 retransmissions after the last successful Msg 1 transmission, and $k$ stands for the number of successful Msg 1 transmissions 


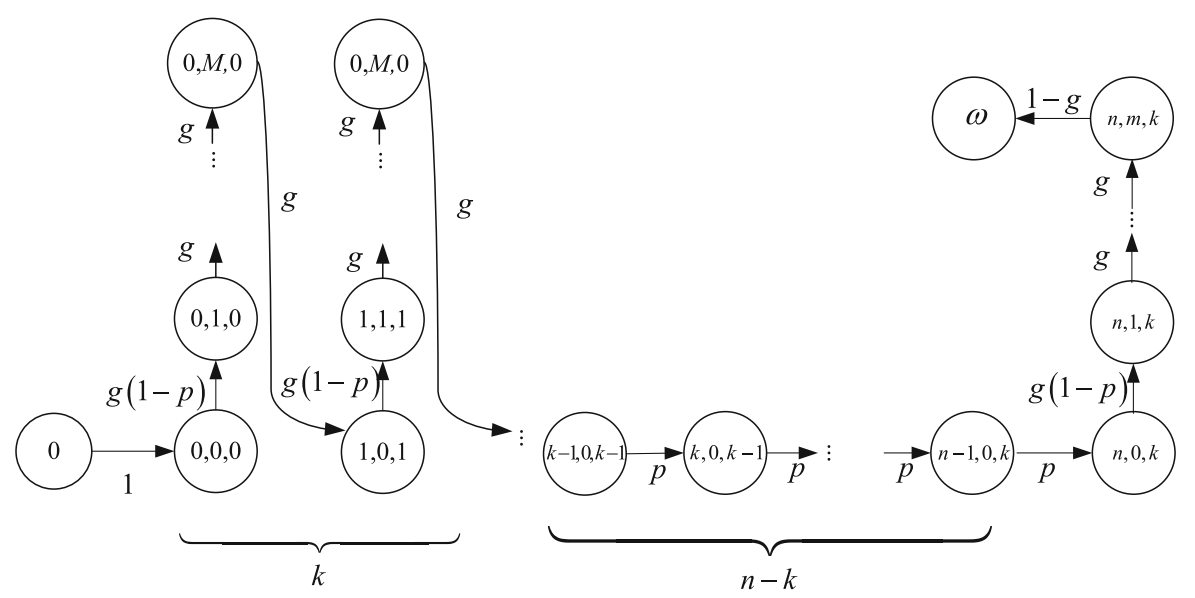

Fig. 3. The example of successful access with Msg 1 and Msg 3 retransmissions

followed by $M+1$ Msg 3 transmissions after each Msg 1 transmission. Figure 3 represents one of possible paths from state $(0,0,0)$ to state $(n, m, k)$ for successful access.

Note, that the access delay for RA procedure is defined as the time interval from the instant when a UE sends its first random access preamble until the UE receives the random access response [7]. In the paper, we focus on the average value $D$ of the access delay. To calculate it we consider all possible scenarios of the RA procedure, i.e. different number of Msg 1 and Msg 3 retransmissions for different combinations of messages' sequences that influence on the overall access delay. For example, in the case of the successful access without any collision the sequence is Msg1 $\rightarrow$ $\mathrm{Msg} 2 \rightarrow \mathrm{Msg} 3 \rightarrow \mathrm{Msg} 4$. For the successful access with two retransmissions of message Msg1 and without Msg3 retransmissions the sequence looks like $\mathrm{Msg} 1 \rightarrow \mathrm{Msg} 1 \rightarrow \mathrm{Msg} 1 \rightarrow \mathrm{Msg} 2 \rightarrow \mathrm{Msg} 3 \rightarrow \mathrm{Msg} 4$.

Note that we do not distinguish between two paths having the same delay between the first RA attempt and the same intermediate state $(n, m, k)$, if the paths differ only Msg 1/Msg 3 positions. For example, the following message sequences (Msg 2 and Msg 4 are omitted) have the equal delays:

$$
\operatorname{Msg} 1 \rightarrow \text { Msg1 } \rightarrow \text { Msg3 } \rightarrow \ldots \rightarrow \text { Msg3 } \rightarrow \text { Msg1 } \rightarrow \text { Msg3 }
$$

and

$$
\operatorname{Msg} 1 \rightarrow \operatorname{Msg} 3 \rightarrow \ldots \rightarrow \text { Msg3 } \rightarrow \text { Msg1 } \rightarrow \text { Msg1 } \rightarrow \text { Msg3 }
$$

Under these assumptions, the probability $P(n, m, k)$ of Markov chain $\left\{\xi_{i}\right\}$ visiting state $(n, m, k)$ when starting from state $(0,0,0)$ is determined by the formula

$$
P(n, m, k)=p^{n-k} C_{n}^{k}\left((1-p) g^{M+1}\right)^{k}(1-p) g^{m},(n, m, k) \in \mathbf{X} .
$$


The first multiplier $p^{n-k}$ stands for $n-k$ Msg 1 collisions, the multiplier $\left((1-p) g^{M+1}\right)^{k}$ stands for $k$ successful Msg 1 transmissions each followed by $M+1$ Msg 3 transmissions, the multiplier $(1-p) g^{m}$ stands for a unique successful Msg 1 transmission followed by $m$ Msg 3 retransmissions, and the binomial coefficient $C_{n}^{k}$ reflects the number of $k$ combinations (successful Msg 1 transmissions) of an $n$ set (Msg 1 retransmissions).

The probabilities of being absorbed in the states $\omega$ and $v$ when starting from state $(0,0,0)$ are

$$
\begin{gathered}
P(\omega)=\sum_{(n, m, k) \in \mathbf{X}} P(n, m, k) \cdot(1-g)=1-\left(p+(1-p) g^{M+1}\right)^{N+1} \\
P(v)=1-P(\omega)=\left(p+(1-p) g^{M+1}\right)^{N+1} .
\end{gathered}
$$

Note, that these probabilities for the RA procedure stand for the access success probability $P(\omega)$ and for the access failure probability $P(v)$.

For successful random access procedure we denote $Q(n, m, k)$ the probability that the RA procedure will be completed right after state $(n, m, k)$, i.e. there will not be any further Msg1/Msg3 collisions. Let $D(n, m, k)$ be the corresponding access delay under the condition that random access procedure is successful.

The access delay $D(n, m, k)$ can be calculated as follows

$$
\begin{aligned}
& D(n, m, k)=(n-k)\left(\Delta_{1}+\Delta_{2}\right)+k\left(\Delta_{1}+\Delta_{3}+M \Delta_{4}\right)+\Delta_{1}+\Delta_{3}+(m+1) \Delta_{4} \\
& =\left(\Delta_{1}+\Delta_{2}\right) \cdot n+\Delta_{4} \cdot m+\left(\Delta_{3}+M \Delta_{4}-\Delta_{2}\right) \cdot k+\Delta_{1}+\Delta_{3}+\Delta_{4} .
\end{aligned}
$$

Form the definition of probability $Q(n, m, k)$ we get the formula

$$
\begin{aligned}
& Q(n, m, k) \\
& =\mathrm{P}\{\text { no Msg1/Msg3 collisions after state }(n, m, k) \mid \text { successful access }\} \\
& =\frac{\mathrm{P}\{\text { no Msg1/Msg3 collisions after state }(n, m, k), \text { successful access }\}}{\mathrm{P}\{\text { successful access }\}} \\
& =\frac{\mathrm{P}\{\text { no Msg1/Msg3 collisions after state }(n, m, k)\}}{\mathrm{P}\{\text { successful access }\}}=\frac{P(n, m, k) \cdot(1-g)}{P(\omega)} .
\end{aligned}
$$

Now, taking into account that the average RA delay, which is calculated only for successfully accessed MTC devices, is determined by the formula

$$
D=\sum_{(n, m, k) \in \mathcal{X}} Q(n, m, k) D(n, m, k),
$$

and taking into account (1)-(5), we finally obtain the formula to calculate the average access delay in closed form 


$$
\begin{aligned}
& D=\left(\Delta_{1}+\Delta_{2}\right) \cdot \frac{C}{(1-p)\left(1-g^{M+1}\right)}\left(1-(N+1) C^{N}+N C^{N+1}\right) \\
& +\Delta_{4} \cdot \frac{1-(M+1) g^{M}+M g^{M+1}}{1-g} \frac{g\left(1-C^{N+1}\right)}{1-g^{M+1}} \\
& +\left(\Delta_{3}+M \Delta_{4}-\Delta_{2}\right) \cdot \frac{g^{M+1}}{1-g^{M+1}}\left(1-(N+1) C^{N}+N C^{N+1}\right) \\
& +\left(\Delta_{1}+\Delta_{3}+\Delta_{4}\right) \cdot\left(1-C^{N+1}\right)
\end{aligned}
$$

where $C=p+g^{M+1}(1-p)$.

The numerical example in the next section illustrates the application of the formulas obtained for calculation the access success probability and the average access delay with given collision probability.

\section{Numerical Example}

We present an example of analysis of a single LTE FDD cell on $5 \mathrm{MHz}$ supporting M2M communications to illustrate some performance measures for RACH with initial data closed to real ones $[7,9,10,18,19]$.

In LTE, the RACH could be configured to occur once every subframe up to once every other radio frame. As in [7] we assume that the PRACH configuration index is equal to 6 , and then for FDD cell we have $1^{\text {st }}$ and $6^{\text {th }}$ subframes of every frame for RACH opportunity, so the RACH occurs every $5 \mathrm{~ms}$, that gives us $200 \mathrm{RACH}$ opportunities per second. The total number of RACH preambles available in LTE is 64 . A number of them are normally reserved for contention free RA procedure (i.e. for intra-system handover or downlink data arrival with lost synchronization), the rest are used for contention based RA procedure. According to [7] we assume that 10 preambles are configured to be dedicated for handovers, therefore, the other 54 can be used contention based random access.

For the scenario with a large number of UEs with RA procedure in the cell and uniformly distributed arrival of $\mathrm{RACH}$ requests the collision probability is given by [9]

$$
p=1-e^{-\gamma /(54 * 200)}
$$

Maximum number of preamble transmission is equal to 10 , hence $N=9$. Maximum number of Msg 3 retransmissions $M=4$ [7]. The terms of the sum in (7) are given below: $\Delta_{1}^{1}=2,5 \mathrm{~ms} ; \Delta_{1}^{2}=1 \mathrm{~ms} ; \Delta_{1}^{3}=2 \mathrm{~ms} ; \Delta_{1}^{4}=5 \mathrm{~ms} ; \Delta_{2}=20 \mathrm{~ms} ; \Delta_{3}=5 \mathrm{~ms}$; $\Delta_{4}=6 \mathrm{~ms}$. The calculation were done for 4 values of the HARQ retransmission probability for Msg 3/Msg $4 \mathrm{~g}=0.02 ; 0.5 ; 0.8 ; 0.95$.

Typically, e.g. [7, 18], RACH performance metrics are analyzed vs the number of MTC devices per cell with maximum of 30000 . In the numerical example we analyze target metrics vs the collision probability $p$, receiving its value from the formula (8) with given random access intensity $\gamma$. Namely the value of $\gamma$ indicates the number of MTC devices in the cell, but it does not reflect the number explicitly. For example, 
$\gamma=25000$ attempts per second corresponds to the case of overload with the collision probability $p$ about 0.9 . By changing the collision probability $p$ from 0 to 1 we compute the access success probability $P(\omega)$ using (2) and the average access delay $D$ using (7).

Figure 4 introduces plots illustrating the access success probability $P(\omega)$ for four values of the HARQ retransmission probability $g$. The plots show that with $g$ less than 0.5 even for $\gamma=10000$ attempts per second $(p=0.6)$ the access success probability is close to 1.

Figure 5 indicates that the average access delay $D$ varies significantly with the changing of the collision probability $p$ and even for minor $g$ can reach values exceeding $160 \mathrm{~ms}$ due to a significant number of preamble retransmissions.

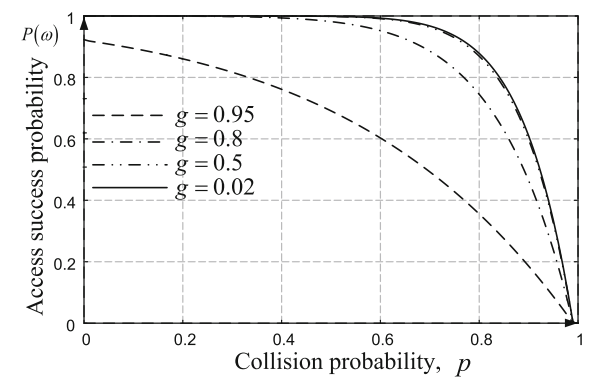

Fig. 4. Access success probability

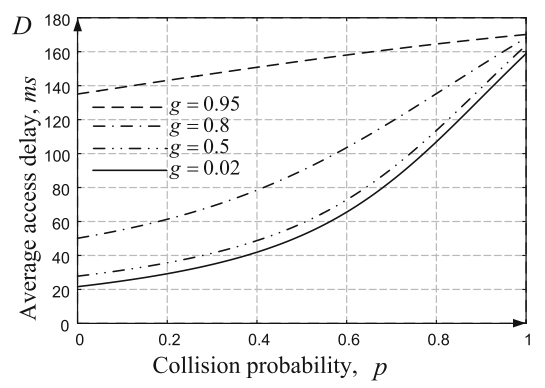

Fig. 5. Average access delay

\section{Conclusion}

In this paper we addressed a RACH procedure for service M2 M traffic in LTE cell and introduced a mathematical model in the form of discrete Markov chain. Note that the access success probability is critical for applications such as fleet management service, when a large number of taxis equipped with fleet management devices gather in a cell, for example near the airport. Another measure, the average access delay, is critical for earthquake monitoring applications, because even tens of milliseconds are very important for an earthquake alarm. The proposed model allows calculating both mentioned performance measures for LTE FDD and TDD cell, UMTS FDD or UMTS 1.28Mcps TDD. 
An interesting task for future study is to derive a formula for the cumulative distribution function (CDF) of the access delay between the first RA attempt and the completion of the random access procedure, for the successfully accessed MTC devices. Another important problem is the construction of analytical models of the overload control mechanisms based on Access Class Barring (ACB) schemes.

Open Access. This chapter is distributed under the terms of the Creative Commons Attribution 4.0 International License (http://creativecommons.org/licenses/by/4.0/), which permits use, duplication, adaptation, distribution and reproduction in any medium or format, as long as you give appropriate credit to the original author(s) and the source, a link is provided to the Creative Commons license and any changes made are indicated.

The images or other third party material in this chapter are included in the work's Creative Commons license, unless indicated otherwise in the credit line; if such material is not included in the work's Creative Commons license and the respective action is not permitted by statutory regulation, users will need to obtain permission from the license holder to duplicate, adapt or reproduce the material.

\section{References}

1. Hasan, M., Hossain, E., Niyato, D.: Random access for machine-to-machine communication in LTE-advanced networks: issues and approaches. IEEE Commun. Mag. 51(6), 86-93 (2013)

2. Condoluci, M., Araniti, G., Dohler, M., Iera, A., Molinaro, A.: Virtual code resource allocation for energy-aware MTC access over 5G systems. Ad Hoc Netw. 43, 3-15 (2016)

3. Gorawski, M., Grochla, K.: Review of mobility models for performance evaluation of wireless networks. In: Gruca, A., Czachórski, T., Kozielski, S. (eds.) Man-Machine Interactions 3. AISC, vol. 242, pp. 573-584. Springer, Heidelberg (2014)

4. Gelenbe, E., Pujolle, G.: Introduction to Queueing Networks. Wiley, New York City (2000)

5. Czachórski, T.: Queueing models for performance evaluation of computer networks transient state analysis. In: Mityushev, V.V., Ruzhansky, M. (eds.) Analytic Methods in Interdisciplinary Applications, vol. 116, pp. 55-80. Springer, Heidelberg (2015). PROMS

6. Andreev, S., Hosek, J., Olsson, T., Johnsson, K., Pyattaev, A., Ometov, A., Olshannikova, E., Gerasimenko, M., Masek, P., Koucheryavy, Y., Mikkonen, T.: A unifying perspective on proximity-based cellular-assisted mobile social networking. IEEE Commun. Mag. 54(4), 108-116 (2016)

7. GPP TR 37.868 - Study on RAN Improvements for Machine-type Communications. Release 11. September 2011 (2011)

8. GPP LTE Release 10 \& beyond (LTE-Advanced)

9. GPP R1-061369: LTE Random-access Capacity and Collision Probability, Ericsson, RAN1\#45, May 2006 (2006)

10. Beale, M.: Future challenges in efficiently supporting M2 M in the LTE standards. In: Proceedings of the 10th Wireless Communications and Networking Conference WCNCW 2012, Paris, France, pp. 186-190. IEEE (2012) 
11. Hossain, M., Niyato, D., Han, Z.: Dynamic Spectrum Access and Management in Cognitive Radio Networks. Cambridge University Press, Cambridge (2009)

12. Borodakiy, V.Y., Buturlin, I.A., Gudkova, I.A., Samouylov, K.E.: Modelling and analysing a dynamic resource allocation scheme for M2 M traffic in LTE networks. In: Balandin, S., Andreev, S., Koucheryavy, Y. (eds.) NEW2AN 2013 and ruSMART 2013. LNCS, vol. 8121, pp. 420-426. Springer, Heidelberg (2013)

13. Buturlin, I.A., Gaidamaka, Y.V., Samuylov, A.K.: Utility function maximization problems for two cross-layer optimization algorithms in OFDM wireless networks. In: Proceedings of the 4th International Congress on Ultra Modern Telecommunications and Control Systems ICUMT-2012, pp. 63-65. IEEE (2012)

14. Gudkova, I., Samouylov, K., Buturlin, I., Borodakiy, V., Gerasimenko, M., Galinina, O., Andreev, S.: Analyzing impacts of coexistence between $\mathrm{M} 2 \mathrm{M}$ and $\mathrm{H} 2 \mathrm{H}$ communication on 3GPP LTE system. In: Mellouk, A., Fowler, S., Hoceini, S., Daachi, B. (eds.) WWIC 2014. LNCS, vol. 8458, pp. 162-174. Springer, Heidelberg (2014)

15. Shorgin, S., Samouylov, K., Gaidamaka, Y., Chukarin, A., Buturlin, I., Begishev, V.: Modeling radio resource allocation scheme with fixed transmission zones for multiservice M2 M communications in wireless IoT infrastructure. In: Nguyen, N.T., Trawiński, B., Kosala, R. (eds.) ACIIDS 2015. LNCS, vol. 9012, pp. 473-483. Springer, Heidelberg (2015)

16. Cheng, M., Lin, G., Wei, H.: Overload control for machine-type-communications in LTE-advanced system. IEEE Commun. Mag. 50(6), 38-45 (2012)

17. Dementev, O., Galinina, O., Gerasimenko, M., Tirronen, T., Torsner, J., Andreev, S., Koucheryavy, Y.: Analyzing the overload of 3GPP LTE system by diverse classes of connected-mode MTC devices. In: Proceedings of the IEEE World Forum on Internet of Things 2014, pp. 309-312 (2014)

18. Wei, C.-H., Bianchi, G., Cheng, R.-G.: Modelling and analysis of random access channels with bursty arrivals in OFDMA wireless networks. IEEE Trans. Wireless Commun. 14(4), 1940-1953 (2015)

19. Borodakiy, V.Y., Samouylov, K.E., Gaidamaka, Y.V., Abaev, P.O., Buturlin, I.A., Etezov, S.A.: Modelling a random access channel with collisions for M2 M traffic in LTE networks. In: Balandin, S., Andreev, S., Koucheryavy, Y. (eds.) NEW2AN/ruSMART 2014. LNCS, vol. 8638, pp. 301-310. Springer, Heidelberg (2014)

20. GPP TS 36.211 - Evolved Universal Terrestrial Radio Access (E-UTRA) - Physical Channels and Modulation (ver. 13.1.0 Release 13 April 2016) (2016) 